\title{
ELEMENTOS DA FORMAÇÁO DO ENFERMEIRO NA PREVENÇÁO DA VIOLÊNCIA CONTRA A PESSOA IDOSA
}

\author{
ELEMENTS OF NURSE TRAINING IN THE PREVENTION \\ OF VIOLENCE AGAINST THE ELDERLY
}

\author{
ELEMENTOS DE LA FORMACIÓN DEL ENFERMERO EN LA \\ PREVENCIÓN DE LA VIOLENCIA CONTRA EL ADULTO MAYOR
}

\author{
Elaiane dos Santos Silva* \\ Eliana Campêlo Lago** \\ Márcia Astrês Fernandes*** \\ Maria Eliete Batista Moura**** \\ Camila Aparecida Pinheiro Landim Almeida*****
}

\begin{abstract}
RESUMO
Objetivo: Analisar elementos da formação do enfermeiro que implicam na atividade profissional quanto à prevenção da violência contra o idoso. Material e Métodos: Pesquisa de abordagem qualitativa realizada com enfermeiros que atuam na Estratégia Saúde da Família (ESF). Os dados foram processados pelo software IRAMUTEQ e analisados por meio da Classificação Hierárquica Descendente com fundamentação no Discurso do Sujeito Coletivo. Resultados: Os resultados destacaram seis classes. Classe 1 - Processo de formação: necessidade de atualização constante, Classe 2 - Dificuldade de identificação dos casos de violência contra o idoso na ESF, Classe 3 - Prevenção da violência contra idosos na ESF, Classe 4 - Reconhecimento de casos de violência contra idosos na ESF, Classe 5 - Identificação precoce de casos de violência e potenciais fatores de risco, Classe 6 - Visita domiciliar ao idoso como estratégia de promoção da saúde e prevenção da violência. Conclusão: A educação permanente desses profissionais a fim de que estejam preparados e orientados para intervençóes nos casos de violência, sendo o enfermeiro um dos principais profissionais deste processo, possibilita a oportunidade de detectar in loco situaçóes de violência contra a pessoa idosa e realizar as devidas intervenções.
\end{abstract}

Palavras chave: Maus-tratos ao Idoso; Enfermeiras e Enfermeiros; Estratégia Saúde da Família; Enfermagem Geriátrica; Educação em Enfermagem.

\footnotetext{
* Enfermeira. Professora do Centro Universitário Uninovafapi. Teresina, PI, Brasil. Email: elaianesantos@hotmail.com

** Enfermeira. Professora do Centro Universitário Uninovafapi. Teresina, PI, Brasil. Email: acadêmico.pro@gmail.com. Autor correspondente.

*** Enfermeira. Professora da Universidade Federal do Piauí - UFPI. Teresina, PI, Brasil. Email: mfernandes@uninovafapi.edu.br

**** Enfermeira. Professora da Universidade Federal do Piauí - UFPI. Teresina, PI, Brasil. Email: liamoura@ufpi.edu.br

***** Enfermeira. Professora do Centro Universitário Uninovafapi. Teresina, PI, Brasil. Email: camila@uninovafapi.edu.br
} 


\begin{abstract}
Objective: To analyze elements of nurses' training that influence their professional activity for the prevention of violence against the elderly. Materials and methods: Qualitative research with nurses working in the Family Health Strategy (ESF) program. The data were processed by the IRAMUTEQ software and analyzed through Descending Hierarchical Classification based on the Discourse of the Collective Subject. Results: The results led to establish six categories: Category 1 - Training process: need for constant updating, Category 2 Difficulty in identifying cases of violence against the elderly in the ESF program, Category 3 - Prevention of violence against the elderly in the ESF, Category 4 - Recognition of cases of violence against the elderly in the ESF, Category 5 - Early identification of cases of violence and potential risk factors, Category 6 - Home care visits to the elderly as a strategy for health promotion and violence prevention. Conclusion: Ongoing training of health professionals is necessary in order for them to be prepared and oriented towards interventions in cases of violence. Being nurses key professionals of this process, they are able to detect situations of violence against the elderly person and to carry out the necessary interventions.
\end{abstract}

Key words: Elder Abuse; Nurses; Family Health Strategy; Geriatric Nursing; Nursing Education.

\title{
RESUMEN
}

Objetivo: Analizar elementos de la formación del enfermero que influyen en su actividad profesional para la prevención de la violencia contra los ancianos. Material y método: Investigación de enfoque cualitativo realizada con enfermeros que se desempeñan en el programa Estrategia Salud de la Familia (ESF). Los datos fueron procesados por el software IRAMUTEQ y analizados por medio de la Clasificación Jerárquica Descendente basada en el Discurso del Sujeto Colectivo. Resultados: Los resultados condujeron a establecer seis clases: Clase 1. Proceso de formación: necesidad de actualización constante, Clase 2. Dificultad de identificación de los casos de violencia contra el anciano en la ESF, Clase 3. Prevención de la violencia contra ancianos en la ESF, Clase 4. Reconocimiento de casos de violencia contra ancianos en la ESF, Clase 5. Identificación precoz de casos de violencia y potenciales factores de riesgo, Clase 6 . Visita domiciliara al anciano como estrategia de promoción de la salud y prevención de la violencia. Conclusión: Es necesaria la formación permanente de los profesionales de la salud a fin de que estén preparados y orientados a intervenciones en los casos de violencia. Siendo el enfermero uno de los principales profesionales de este proceso, posibilita la oportunidad de detectar in situ situaciones de violencia contra la persona anciana y realizar las debidas intervenciones.

Palabras clave: Maltrato al Anciano; Enfermeros; Estrategia de Salud Familiar; Enfermería Geriátrica; Educación en Enfermería.

Fecha recepción: 21/06/2018 Fecha aceptación: 15/01/2019

\section{INTRODUÇÃO}

A violência contra o idoso é tanto um óbice ao desenvolvimento da saúde pública, quanto um problema progressivo para o campo ${ }^{(1)}$. Evidências demonstram que os tipos de violência variam desde psicológicos a agressóes físicas ${ }^{(2)}$. Ao tempo que indicador de progresso nacional tem se ampliado, como o aumento na expectativa de vida e da população idosa no Brasil nos últimos $\operatorname{anos}^{(3,4)}$, esses números acompanham o acréscimo na violência contra esse grupo social.

O impacto da violência contra o idoso no setor da saúde pode ser expresso em números, conforme dados de 2008 da Organização Mundial da Saúde$\mathrm{OMS}^{(5)}$. Um total de 20.303 idosos morreu por 
causas externas (acidentes e violência), significando $3,1 \%$ das mortes dessas pessoas no Brasil. Nesse mesmo ano, 65,4\% dos mortos por essas causas precisaram de atendimento e faleceram quando ainda estavam sendo atendidos em uma unidade de saúde ${ }^{(6)}$. Em 2009, ano seguinte, as agressóes totalizaram 1.929 óbitos, de um total de 21.453 fatalidades por causas externas ${ }^{(7)}$.

A Organização Mundial de Saúde (OMS) conceitua maus-tratos à pessoa idosa como ato único ou repetido, ou ainda, como ausência de ação apropriada que ocasione dano, sofrimento ou angústia, e que ocorra dentro de um relacionamento de confiança. A violência e a violação dos direitos humanos são consideradas um fenômeno biopsicossocial ${ }^{(8)}$. O Ministério da Saúde Brasileiro classifica a violência contra a pessoa idosa em física, sexual, psicológica, econômica, institucional, abandono/negligência e autonegligência ${ }^{(9)}$. Ademais, a violência contra o idoso pode ser objetivada de diferentes formas: danos físicos, mentais e morais ${ }^{(10)}$.

No Brasil, as violências contra os idosos também se expressam sob a forma de discriminação, considerando idosos como "descartáveis e um peso social"(11). A situação é decorrente do preconceito cultural queainda persistecontra o idoso no Brasil ${ }^{(12)}$, principalmente em contextos sociais e de saúde, tornando necessária a capacitação de profissionais na prevenção, identificação e tratamento de maustratos em idosos, uma vez que os serviços de saúde em geral, e mais particularmente os setores de emergência e os ambulatórios, constituem uma das principais portas de entrada das vítimas de maus$\operatorname{tratos}^{(13)}$.

A preocupação com a formação dos profissionais de enfermagem emerge desta necessidade latente de capacitação e do vínculo deste ao atendimento, direto às pessoas idosas vítimas de violência que procuram serviços de saúde. Sua inserção na equipe multiprofissional da Estratégia Saúde da FamíliaESF, no desempenho de atividades de promoção, prevenção, diagnóstico e tratamento, por meio de buscas ativas em visitas domiciliares, atendimentos em hospitais, postos de saúde, ambulatórios e home care, que oportuniza a convivência próxima com o público em questão. Por este convívio e contato, os enfermeiros podem atuar na notificação e percepção do caso de violência, situaçôes que podem auxiliar na descontinuidade dos atos de violência ${ }^{(10,11)}$. Contudo, o gap entre formação e atuação profissional pode apresentar óbices na integração entre aquilo que é aprendido na graduação e a prestação de serviços ${ }^{(14)}$. Estas dificuldades estão relacionadas, em suma, com competências e habilidade técnica e a distância prática entre a formação acadêmica e a realidade do profissional ${ }^{(14)}$.

O estudo teve como objetivo analisar elementos da formação do enfermeiro que implicam na atividade profissional quanto à prevenção da violência contra o idoso.

\section{MATERIAL E MÉTODO}

Pesquisa de abordagem qualitativa fundamentada no Discurso do Sujeito Coletivo ${ }^{(15)}$ realizada com 24 enfermeiros de equipes de saúde da família de um município do interior do Maranhão.

Foram incluídos profissionais que atuavam em alguma equipe da Estratégia da Saúde da Família por, no mínimo, um ano e com atuação exclusiva na zona urbana da cidade, convidados a participar do estudo. A inclusão dos participantes foi concluída por critérios de saturação teórico-empírica, quando as respostas não traziam mais contribuições significantes ou estas eram mínimas ${ }^{(16)}$.

A coleta dos dados ocorreu durante o ano de 2015, com entrevistas individuais guiadas por um roteiro semiestruturado incluindo tópicos sobre o cuidado na prevenção da violência contra o idoso. A entrevista foi realizada em sala reservada com duração média de 40 minutos.

As entrevistas foram transcritas na integra e montou-se um corpus textual que compreendeu o texto do to taldeentrevistas. Parao processamento dos dados utilizou-se o software IRAMUTEQ (Interface de $\mathrm{R}$ pour les Analyses Multidimensionnelles de Texte set et de Questionnaires) que possibilita a organização do corpus textual e diferentes formas de análises estatísticas sobre este ${ }^{(17)}$. Para a análise do texto, seguiu-se a Classificação Hierárquica Descendente.

O uso de softwares em análise de dados qualitativos tem sido crescente por visar redução do trabalho operacional com a organização dos dados e ampliar as possibilidades do pesquisador quanto sua análise ${ }^{(18,19)}$. O texto das entrevistas foi transcrito para a construção do corpus e organizado em um 
único arquivo de texto, conforme orientações do tutorial do IRAMUTEQ ${ }^{(19)}$. O corpus foi formado pelo conjunto de textos a ser analisado.

A pesquisa foi aprovada pelo Comitê de Ética e Pesquisa do Centro Universitário UNINOVAFAPI, sob parecer no 879.609 .

\section{RESULTADOS}

O IRAMUTEQ reconheceu a separação do corpus em 101 unidades de texto elementares (UCE) divididas em 121 segmentos de texto, com 83,47\% de aproveitamento do material.

O material analisado resultou em seis classes. Classe 1-Processo de formação: necessidade de atualização constante, Classe 2-Dificuldade de identificação dos casos de violência contra o idoso na ESF, Classe 3-Prevenção da violência contra idosos na ESF, Classe 4-Reconhecimento de casos de violência contra idosos na ESF, Classe 5-Identificação precoce de casos de violência e potenciais fatores de risco, Classe 6-Visita domiciliar ao idoso como estratégia de promoção da saúde e prevenção da violência, conforme se observa na Figura 1.

A relação entre as classes demonstra que a formação do enfermeiro possui reflexos nas suas atitudes e percepçóes em relação à prevenção da violência contra a pessoa idosa atendida na Estratégia Saúde da Família. Esta formação é "complementada" por cursos e programas de capacitação profissional, gerando reflexos no trabalho em equipe.

Classe 1: Processo de formação: necessidade de atualizaçáo constante

Os enfermeiros elencam déficit no processo de formação pela ausência de elementos que enfoquem a violência contra a pessoa idosa. Este viés de formação acarreta falta de habilidade para lidar com a problemática no quotidiano dos serviços da estratégia saúde da família, observa-se:

Formação não adequada e não foca na violência ao idoso, falta de capacitaçáo dos profissionais e a própria dificuldade dos profissionais em lidar com esses tipos de situação. Ent. 02
Faz-se necessário intervir com competência e habilidade e os enfermeiros apresentam lacunas em seu processo de formação. Além disso, em aspectos gerenciais, o planejamento das equipes é afetado por falta de proximidade com o tema:

A temática é trabalhada diante da necessidade ou sugestão feita pela equipe não para desenvolver açóes e atividades abordando essa questão, acho que seria de suma relevância a realização de capacitação aos profissionais de saúde com abrangência em todos os setores... outra sugestão interessante seria a criação das semanas de combate a esse tipo de violência assim poderíamos tratar de maneira incisiva dentro do município. Ent. 05

O trabalho de toda a equipe é prejudicado, o discurso dos enfermeiros desvela a adoção de medidas que são delegadas para outros integrantes da equipe de saúde. Tal delegação é realizada de forma arbitrária uma vez que o profissional enfermeiro não tem capacitação para lidar com os possíveis casos que possam surgir no serviço:

Não tenho capacitação. O que é feito para a prevenção são palestras e busca ativa com o apoio dos ACS. Ent. 17

Nesta classe, observa-se que o trabalho em equipe tem prejuízos na qualidade do funcionamento em decorrência da formação do enfermeiro que não contempla de forma satisfatória a complexidade da violência contra o idoso.

Classe 2: Dificuldade de identificação dos casos de violência contra o idoso na ESF

Um dos passos iniciais para a gestão de casos de violência contra o idoso na ESF é o reconhecimento e identificação do caso. Um processo de formação bem conduzido e que aborde o tema com profundidade prepara profissionais capazes de identificar e conduzir um caso de violência.

Uma das principais dificuldades é identificar a violência, uma vez que a maioria das vezes pessoas só considera violência a física, mas é bem comum a exploração financeira e do trabalho. Ent. 08 
Reconhecer as peculiaridades de atos de violência praticados contra um idoso requer uma formação fundamentada em aporte teórico sólido que possibilite o enfermeiro ter raciocínio crítico e reflexivo sobre os fatos que se apresentam em seu cotidiano de trabalho junto à comunidade.

Figura 1. Dendograma Formação do enfermeiro na prevenção da violência contra a pessoa idosa na estratégia saúde da família.

\begin{tabular}{|c|c|c|c|c|}
\hline & Palavra & $\chi^{2}$ (Qui-quadrado) & Palavra & $\overline{\chi^{2} \text { (Qui-quadrado) }}$ \\
\hline $\begin{array}{c}\text { Classe } 1 \\
15 \text { UCE - 14,85\% } \\
\text { Processo de formação: } \\
\text { necessidade de } \\
\text { atualização constante }\end{array}$ & $\begin{array}{c}\text { Serviço } \\
\text { Capacitação } \\
\text { Sugestão } \\
\text { Problema } \\
\text { Falta } \\
\text { Planejamento } \\
\text { Município } \\
\text { Social }\end{array}$ & \begin{tabular}{r|}
23,88 \\
23,88 \\
23,66 \\
17,66 \\
13,54 \\
11,92 \\
11,92 \\
8,48
\end{tabular} & $\begin{array}{c}\text { Necessidade } \\
\text { Referenciar } \\
\text { Adequado } \\
\text { Realização } \\
\text { Desenvolver } \\
\text { Órgão } \\
\text { ACS } \\
\text { Saúde }\end{array}$ & $\begin{array}{l}6,56 \\
6,56 \\
6,56 \\
6,56 \\
6,56 \\
6,23 \\
4,53 \\
2,89\end{array}$ \\
\hline $\begin{array}{c}\text { Classe 2 } \\
16 \text { UCE - 15,84\% } \\
\text { Dificuldade de } \\
\text { identificação dos casos } \\
\text { de violência contra o } \\
\text { idoso na ESF }\end{array}$ & $\begin{array}{c}\text { Dificuldade } \\
\text { Ocorrer } \\
\text { Trabalho } \\
\text { Encontrar } \\
\text { Necessário } \\
\text { Caso } \\
\text { Unidade } \\
\text { Atendimento } \\
\text { Assim }\end{array}$ & $\begin{array}{c}19,14 \\
17,43 \\
12,36 \\
12,36 \\
11,11 \\
10,93 \\
9,62 \\
7,69 \\
7,69\end{array}$ & $\begin{array}{l}\text { Quando } \\
\text { Evitar } \\
\text { Educativo } \\
\text { Demanda } \\
\text { ESF } \\
\text { Órgão } \\
\text { Situação } \\
\text { Próprio }\end{array}$ & $\begin{array}{l}6,06 \\
5,99 \\
5,99 \\
5,99 \\
5,8 \\
5,58 \\
4,86 \\
3,65\end{array}$ \\
\hline $\begin{array}{c}\text { Classe } 3 \\
20 \text { UCE - 19,8\% } \\
\text { Prevenção da violência } \\
\text { contra idosos na ESF }\end{array}$ & $\begin{array}{c}\text { Formação } \\
\text { Violência } \\
\text { Vitimar } \\
\text { Capacitar } \\
\text { Disciplina } \\
\text { Questão } \\
\text { Prevenção } \\
\text { Possuir } \\
\text { Direito }\end{array}$ & $\begin{array}{c}44,29 \\
17,18 \\
16,21 \\
12,52 \\
7,99 \\
6,6 \\
5,44 \\
5,35 \\
4,99\end{array}$ & $\begin{array}{c}\text { Comum } \\
\text { Não } \\
\text { Contribuir } \\
\text { Enfermagem } \\
\text { Sinal } \\
\text { Família } \\
\text { Conhecimento } \\
\text { Aspecto }\end{array}$ & \begin{tabular}{l|l}
4,28 \\
3,8 \\
3,78 \\
3,66 \\
3,66 \\
2,85 \\
2,52 \\
2,39
\end{tabular} \\
\hline $\begin{array}{c}\text { Classe } 4 \\
13 \text { UCE- 12,87\% } \\
\text { Reconhecimento de } \\
\text { casos de violência } \\
\text { contra idosos na ESF }\end{array}$ & $\begin{array}{c}\text { Paciente } \\
\text { ESF } \\
\text { Momento } \\
\text { Atar } \\
\text { Atuar } \\
\text { Receber } \\
\text { Ano } \\
\text { Desenvolvido } \\
\text { Atuação }\end{array}$ & \begin{tabular}{r|}
23,88 \\
23,66 \\
17,66 \\
13,54 \\
11,92 \\
11,92 \\
8,48 \\
6,56 \\
6,56 \\
\end{tabular} & $\begin{array}{c}\text { Tema } \\
\text { Praticar } \\
\text { Observação } \\
\text { Vivenciar } \\
\text { Muito } \\
\text { Quando } \\
\text { Prevenção } \\
\text { Sofrer }\end{array}$ & $\begin{array}{l}6,56 \\
6,56 \\
6,56 \\
6,23 \\
6,23 \\
4,53 \\
2,89 \\
3,45\end{array}$ \\
\hline $\begin{array}{c}\text { Classe } 5 \\
17 \text { UCE - 16,83\% } \\
\text { Identificação precoce } \\
\text { de casos de violência } \\
\text { e potenciais fatores de } \\
\text { risco }\end{array}$ & $\begin{array}{c}\text { Físico } \\
\text { Informar } \\
\text { Mostrar } \\
\text { Cuidado } \\
\text { Dever } \\
\text { Ao } \\
\text { Cuidador } \\
\text { Trabalhar } \\
\text { Assunto }\end{array}$ & \begin{tabular}{l|l}
16,02 \\
15,28 \\
14,99 \\
14,6 \\
12,77 \\
12,77 \\
12,54 \\
10,58 \\
10,07
\end{tabular} & $\begin{array}{l}\text { Dispensar } \\
\text { Observar } \\
\text { Bem } \\
\text { Praticar } \\
\text { Presente } \\
\text { Grupo } \\
\text { Vida } \\
\text { Através }\end{array}$ & $\begin{array}{c}10,07 \\
7 \\
7 \\
5,8 \\
5,49 \\
5,01 \\
5,01 \\
3,27\end{array}$ \\
\hline $\begin{array}{c}\text { Classe } 6 \\
20 \text { UCE - 19,8\% } \\
\text { Visita domiciliar ao } \\
\text { idoso como estratégia } \\
\text { de promoção da saúde e } \\
\text { prevenção da violência }\end{array}$ & $\begin{array}{c}\text { Visita } \\
\text { Passar } \\
\text { Domiciliar } \\
\text { Neto } \\
\text { Cuidar } \\
\text { Aposentadoria } \\
\text { Acamado } \\
\text { Só } \\
\text { Também }\end{array}$ & $\begin{array}{r}29,9 \\
21,3 \\
17,6 \\
16,8 \\
16,8 \\
12,5 \\
12,5 \\
8,8 \\
7,9\end{array}$ & $\begin{array}{c}\text { Sempre } \\
\text { Dia } \\
\text { Tomar } \\
\text { Orientar } \\
\text { Cuidador } \\
\text { Procurar } \\
\text { Resolver } \\
\text { Medo }\end{array}$ & $\begin{array}{l}5,3 \\
5,3 \\
5,3 \\
5,3 \\
5,1 \\
4,2 \\
4,2 \\
4,2\end{array}$ \\
\hline
\end{tabular}

Nota: As classes da CHD são descritas pelos vocábulos com maior associação com a classe (Qui-quadrado - $\chi^{2}$ ), em teste de associação da palavra à classe com significância. 
Classe 3: Prevenção da violência contra idosos na ESF

A prevenção da violência deve ser trabalhada junto à comunidade na desmistificação do próprio processo de envelhecimento, devendo ser trabalhada a compreensão do idoso sobre si, sobre seu papel junto à família e sua função social enquanto sujeito inserido em um contexto.

Alguns dos aspectos que interferem na prestação da assistência a pessoa idosa vitima de violência são por parte do idoso: a dependência em todas as suas formas como física mental afetiva socioeconômica medo da vitima de possiveis represálias. Sentimento de culpa, a pessoa idosa pode pensar que é sua a culpa por estar sofrendo maus tratos, pois não foi um bom pai ou uma boa máe e agora está colhendo os resultados. Ent. 07

O sentimento de culpa pode interferir na identificação dos casos, mas o enfermeiro deve estar com suporte teórico e prático suficiente para a condução dos casos, especialmente no desenvolvimento de intervençóes capazes de promover prevenção comunitária abrangente que envolva públicos de diferentes idades.

\section{Classe 4: Reconhecimento de casos de violência contra idosos na ESF}

Nesta classe é relatada a dificuldade de identificação dos casos, especialmente pela omissão do idoso e por medo do desfecho. Entretanto, a detecção de fatores de risco é uma iniciativa para prevenção, detecção precoce e promoção de condutas apropriadas para evitar esse tipo de violência. Além disso, o vínculo entre profissional de saúde e usuário do serviço é um elemento fundamental para gerar confiança capaz de construir um caminho de confiança no serviço e no profissional de saúde.

Felizmente até o presente momento ainda não vivenciei. Um obstáculo que muitas vezes pode acontecer é a omissão. Muitos idosos são violentados até mesmo por seus familiares, mas possuem receio de procurar ajuda e confiança a quem procurar. Ent. 12

Classe 5: Identificação precoce de casos de violência e potenciais fatores de risco
A identificação de fatores de risco pode ser uma estratégia adotada pelas equipes de saúde para a redução dos casos de violência. Considerando que a maior parte dos casos ocorre no ambiente familiar, os participantes da pesquisa informaram que com base em sua atuação nas ESF, os cuidadores não estão preparados para cuidar de uma pessoa idosa, como se pode observar:

O convivio familiar estressante e cuidadores despreparados ou sobrecarregados tendem a agravar a situação do idoso, por acompanhar rigorosamente, através de visitas domiciliares, observar qualquer sinal de irregularidade como violência psicológica, física, violência econômica, financeira e patrimonial. Ent. 04

Diante de ser muito especifico à atenção a este grupo pude observar e vivenciar que a graduação não é abordado sobre o tema de forma mais ampla. Ent. 24

Dada a complexidade do tema, a prevenção dos casos de violência vai além de práticas usuais como palestras sobre o tema, evidencia-se a necessidade de uma abordagem mais ampla, incluindo a capacitação de cuidadores para o cuidado adequado ao idoso.

Classe 6: Visita domiciliar ao idoso como estratégia de promoção da saúde e prevençáo da violência

Avisita domiciliar é apresentada pelos participantes como uma importante ferramenta de promoçáo da saúde e prevenção da violência contra o idoso. São apresentados os cuidados na visita domiciliar:

Faço palestras e orientação aos cuidadores, um casal de idosos que moravam sozinhos em uma casa, praticamente sem nada, passava fome porque a filha era drogada e só ia visita-los no dia da aposentadoria... a maior dificuldade é acionar as pessoas certas e náo ter o retorno de resolução do caso. Ent. 06

O discurso dos enfermeiros participantes desta pesquisa elucida uma questão de extrema relevância para a enfermagem atual: o processo de formação do enfermeiro voltado para problemas presentes no cotidiano de comunidades envolvendo um grupo cada vez maior, os idosos. A 
violência contra a pessoa idosa deve ser enfrentada por profissionais com suporte teórico e prático que possibilite o manejo adequado dos casos, fundamentado em evidências científicas.

\section{DISCUSSÃO}

Há necessidade de capacitação dos profissionais de saúde para a identificação e intervenção com competência nos casos de violência sofrida por idosos. Entende-se que o processo deve ser iniciado na graduação e continuar durante a atuação profissional nos serviços de saúde por meio de cursos de atualização e capacitação. Em suma, há uma lacuna entre a formação desenvolvida na graduação e a prática do profissional ${ }^{(14)}$.

No contato direto com os idosos que usam os serviços de saúde, a investigação e identificação dos casos de violência e maus-tratos cabem ao profissional de saúde, bem como a ação coordenada com outros profissionais para traçarem intervençôes capazes de gerar efeitos positivos nos indicadores de violência ${ }^{(5,10,12)}$.

A tarefa dos profissionais junto às vitimas de maus tratos é obstaculada porque, além de acolher e detectar os casos, é preciso acompanhar as vítimas, sendo necessária uma formação sólida. A falta de aporte teórico e habilidade prática para lidar com situações de violência estão relacionas ao sentimento de impotência, que surge quando o profissional se depara com casos e não se sente suficientemente capacitado para abordá-lo. O medo da exposição e de represálias por parte do agressor é latente ${ }^{(20)}$.

Estudos têm reconhecido que os profissionais de saúde são considerados essenciais na identificação de indivíduos e grupos populacionais de risco para a violência, pela posição que ocupam na prestação de serviços à população, e para a promoção e prevençãao ${ }^{(21)}$. A crescente necessidade de atendimento às vítimas de violência que chegam aos serviços de saúde evidencia precariedade na estrutura do sistema de saúde e dificuldades de seus profissionais no atendimento e acompanhamento dessas pessoas, contribuindo para a reincidência e agravamento de $\operatorname{casos}^{(3,4)}$. Muitos destes problemas estão relacionados ao processo de formação e capacitação dos membros das equipes de saúde ${ }^{(21)}$.

Evidências internacionais indicam a comple- xidade dos casos que podem ser registrados desde o ambiente familiar doméstico a casas de repouso e contribuem para a fragilidade do envelhecimento. Além disso, a violência contra o idoso apresenta viés de gênero, um estudo latino-americano mostrou evidências de que as idosas são mais atingidas ${ }^{(22-25)}$.

A capacitação dos recursos humanos e a orientação dos cuidadores/familiares certificando a qualidade $\mathrm{da}$ atenção oferecida; garante a obrigação da notificação dos casos suspeitos ou confirmados de violência aos órgãos públicos e as ações de prevenção desses agravos ${ }^{(6)}$. Contudo, os índices de agressóes e demais tipos de violência externa continuam aumentando ${ }^{(1)}$, bem como a não consideração de outros agravos, que não o tipo físico e visualmente mais fácil de ser identificado ${ }^{(9)}$.

A maior parte dos estudos que abordam a questão da capacitação de profissionais da saúde em relação à violência indica a necessidade de se desenvolver estratégias para prepará-los melhor sobre o tema, ainda indicando a formação como incompleta ou carente em alguns aspectos ${ }^{(14)}$. Além da necessidade de investir na conscientização dos profissionais sobre a violência e de recursos disponíveis para a sua abordagem, indicam também a necessidade de introduzir formalmente uma disciplina nos currículos e desenvolver programas de capacitação permanente das equipes de saúde ${ }^{(21)}$.

Além de a violência contra os idosos e suas manifestaçóes serem difíceis de ser reconhecidas, esse problema não tem se apresentado como uma prática de relevância social e acadêmica. Neste momento histórico, em que a quantidade de idosos tem crescido, torna-se um tema obrigatório da pauta de questóes sociais ${ }^{(1)}$.

Os serviços de atenção primária são estratégicos para lidar com casos de violência familiar, funcionando como a porta de entrada dos serviços públicos de saúde. A literatura da última década informa que os profissionais já vêm incorporando em suas rotinas as determinações de políticas públicas direcionadas ao enfrentamento da violência familiar ${ }^{(21)}$.

\section{CONCLUSÃO}

Neste estudo, evidenciou-se que, dentre os elementos formativos ou sua ausência, não é habitual que os profissionais recebam treinamentos 
ou capacitações para atuar na prevenção da violência contra a pessoa idosa na Estratégia Saúde da Família, com principal dificuldade a identificação destas agressóes ou abusos. A proximidade da relação social entre idosos e agressores contribui para esta dificuldade e, muitas vezes, aqueles que sofrem não denunciam os maus-tratos a que estão sujeitos.

Como a unidade básica de saúde é porta de entrada do serviço de saúde para a população brasileira em geral, torna-se necessário a valorização de estratégias de educação permanente e continuada, visando suprir déficits de formação. Neste caso específico, os múltiplos aspectos que compóem o tema violência.

As limitaçóes do estudo dizem respeito ao lócus da pesquisa, por enfocar a realidade de um município no interior do país, o que, por outro lado, pode auxiliar a compreensão da questão em outras localidades que, por estarem em condições similares, podem possuir características também análogas. Pesquisas futuras são recomendadas e podem explorar contextos de grandes centros urbanos brasileiros a fim de contribuir com mais evidências. Desta forma, comparações podem ser feitas sobre o mesmo fenômeno em realidades distintas.

\section{REFERENCIAS}

1. Santana IO, Vasconcelos DC, Coutinho MPL. Prevalência da violência contra o idoso no Brasil: revisão analítica. Arq. bras. psic. [Internet]. 2016 [citado 22 Maio 2017]; 68(1): 126-139. Disponível em: http:// pepsic.bvsalud.org/scielo.php?script=sci_ arttext\&pid=S1809-52672016000100011

2. Giraldo-Rodríguez L, Rosas-Carrasco O, MinoLeón D. Abuse in Mexican Older Adults with Long-Term Disability: National Prevalence and Associated Factors. J Am Geriatr Soc [Internet]. 2015 [cited 2019 Jan 22]; 63(8): 1594-600. Available from: https://www.ncbi.nlm.nih.gov/ pubmed/26289685

3. Bolsoni CC, Conceição TB, Lindner SR, Coelho EBS. Violência contra o idoso: uma meta-síntese. Saúde Transform. Soc [Internet]. 2016 [citado 02 Jul 2017]; 8(1): 98-105. Disponível em: http:// www.redalyc.org/pdf/2653/265346077012.pdf

4. Kernkamp Cda L, Costa CK, Massuda EM, Silva ES, Yamaguchi MU, Bernuci MP. Perfil de morbidade e gastos hospitalares com idosos no Paraná, Brasil, entre 2008 e 2012. Cad Saude Publica [Internet]. 2016 [citado 18 Jan 2017]; 32(7): 1-14. Disponível em: http://www.scielosp.org/pdf/ csp/v32n7/1678-4464-csp-32-07-e00044115.pdf

5. Araújo LF, Cruz EA, Rocha RA. Representaçóes sociais da violência na velhice: estudo comparativo entre profissionais de saúde e agentes comunitários de saúde. Psicol. Soc. [Internet]. 2013 [citado 13 Abr 2017]; 25(1): 203-212. Disponível em: http://www.scielo.br/scielo.php?pid=S010271822013000100022\&script=sci_abstract\&tlng=pt

6. Ribeiro AP, Souza ER, Valadares FC. Atendimento de saúde para pessoas idosas vítimas de violência no município do Rio de Janeiro. Cien Saude Colet. 2012; 17(5): 1167-177.

7. Mascarenhas MDM, Andrade SSCA, Neves ACM, Pedrosa AAG, Silva MMA, Malta DC. Violência contra a pessoa idosa: análise das notificaçôes realizadas no setor saúde - Brasil, 2010. Cien Saude Colet. 2012; 17(9): 2331-341.

8. Sousa DJ, White HJ, Soares LM, Nicolosi GT, Cintra FA, D'elboux MJ. Maus-tratos contra idosos: atualização dos estudos brasileiros. Rev. Bras. Geriatr. Gerontol. 2010;13(2): 321-28.

9. Brasil. Ministério da Saúde. Secretaria de Atenção à Saúde. Departamento de Atenção Básica. Caderno de atenção básica n. 19: Envelhecimento e saúde da pessoa idosa. Brasília (DF): Ministério da Saúde; 2006. p. 192.

10. Minayo MCS, Souza ER. As múltiplas mensagens da violência contra idosos. Rio de Janeiro: Fiocruz; 2003. p. 1223-42.

11. Souza JAV, Freitas MC, Queiroz TA. Violência contra os idosos: análise documental. Rev Bras Enferm. 2007; 60(3): 268-272.

12. Couto MCPDP, Koller SH, Novo R, SanchezSoares P. Avaliação de discriminação contra idosos em contexto brasileiro-ageismo. Psic.: Teor. e Pesq. [Internet] 2009 [citado 23 Maio 2017]; 25(4): 509518. Disponível em: http://www.scielo.br/pdf/ptp/ v25n4/a06v25n4.pdf

13. Freitas EV, Ligia PY; Cançado FAX, Johannes D, Gorzoni ML. Tratado de geriatria e gerontologia. $2^{\text {a }}$ ed. Rio de Janeiro: Guanabara \& Koogan; 2006.

14. Souza LP, Silva WSS, Mota ÉC, Freitas Santana JM, Graças Siqueira L, Oliveira Silva CS, et al. Os desafios do recém-graduado em Enfermagem no mundo do trabalho. Rev Cubana Enfermer [Internet]. 2015 [citado 12 Maio 2017]; 30(1). Disponível em: http://www.revenfermeria.sld.cu/ index.php/enf/article/view/127

15. Lefèvre F, Lefèvre AM. O discurso do sujeito coletivo: um novo enfoque em pesquisa qualitativa; desdobramentos. São Paulo: EDUCS; 2005. 
16. Minayo MCS. Amostragem e saturação em pesquisa qualitativa: consensos e controvérsias. Rev. Pesqui. Qual. [Internet]. 2017 [citado 28 Maio 2017]; 5(7):1-12. Disponível em: http://rpq.revista. sepq.org.br/index.php/rpq/article/view/82/59

17. Camargo BV, Justo AM. Tutorial para uso do software de análise textual IRAMUTEQ [Internet]. Florianópolis: Laboratório de Psicologia Social da Comunicação e Cognição- LACCOS - Universidade Federal de Santa Catarina; 2016 [citado 8 Maio 2017]. Disponível em: http://www. iramuteq.org/documentation/fichiers/Tutorial\%20 IRaMuTeQ\%20em\%20portugues_17.03.2016.pdf

18. Mesquita RF, Matos FRN. A abordagem qualitativa nas ciências administrativas: aspectos históricos, tipologias e perspectivas futuras. Revista Brasileira de Administração Científica [Internet]. 2014 [citado 23 Maio 2017]; 5(1): 7-22. Disponível em: http://www.sustenere.co/index.php/rbadm/article/ view/SPC2179-684X.2014.001.0001

19. Mesquita RF, Sousa MB, Martins TB, Matos FRN. Óbices metodológicos da prática de pesquisa nas ciências administrativas. Revista Pensamento Contemporâneo em Administração [Internet]. 2014 [citado 18 Mayo 2017]; 8(1): 5065. Disponível em: https://search.proquest.com/op enview/1b1c3886f669f3e8b903577ca4972c86/1? pq-origsite $=$ gscholar $\&$ cbl $=2032639$
20. Wanderbroocke ACNS, More CLOO. Significados de violência familiar contra o idoso na perspectiva de profissionais da Atenção Primária à Saúde. Cien Saude Colet. 2012; 17(8): 2095-2103.

21. Souza ER, Ribeiro AP, Penna LHG, Ferreira AL, Santos NC, Tavares CMM. O tema violência intrafamiliar na concepção dos formadores dos profissionais de saúde. Cien Saude Colet. 2009; 14:(5): 1709-1719.

22. Gomes CS, Pirkle CM, Zunzunegui MV, Guedes DT, Barbosa JFDS, Hwang P, et al. Frailty and life course violence: The international mobility in aging study. Arch Gerontol Geriatr. 2018; 76: 26-33.

23. Guedes DT, Curcio CL, Llano BA, Zunzunegui $\mathrm{MV}$, Guerra R. The gender gap in domestic violence in older adults in Latin America: the IMIAS Study. Rev Panam Salud Publica. 2015; 37(4): 293-300.

24. Levine M. Sex and the nursing home. Am J Orthopsychiatry. 2016; 86(3): 355-359.

25. Guedes DT, Vafaei A, Alvarado BE, Curcio CL, Guralnik JM, Zunzunegui MV, et al. Experiences of violence across life course and its effects on mobility among participants in the International Mobility in Aging Study. BMJ open [Internet]. 2016 [cited 2019 Jan 22]; 6(10): e012339. Available from: https://www.ncbi.nlm.nih.gov/ pubmed/27737884 